\title{
Compression of Tumors Causes Drug Resistance
}

\section{Pressure that develops as a tumor grows can limit the effectiveness of chemotherapy treatments.}

\author{
By Dan Garisto
}

C ancerous tumors can be resistant to chemotherapy drugs as a result of pressure on the cells, according to new experiments [1]. Researchers found that clumps of cancer cells that were compressed during growth were less affected by cancer drugs than uncompressed clumps. Many chemotherapy drugs, including those tested, target cells that are rapidly reproducing, or "proliferating." The proliferation rate is reduced by compression, which explains the observed drug resistance. The researchers used their results to create a theoretical model that links drug resistance to the effects of compression. Understanding the relationship between compressive forces and drug resistance could help researchers

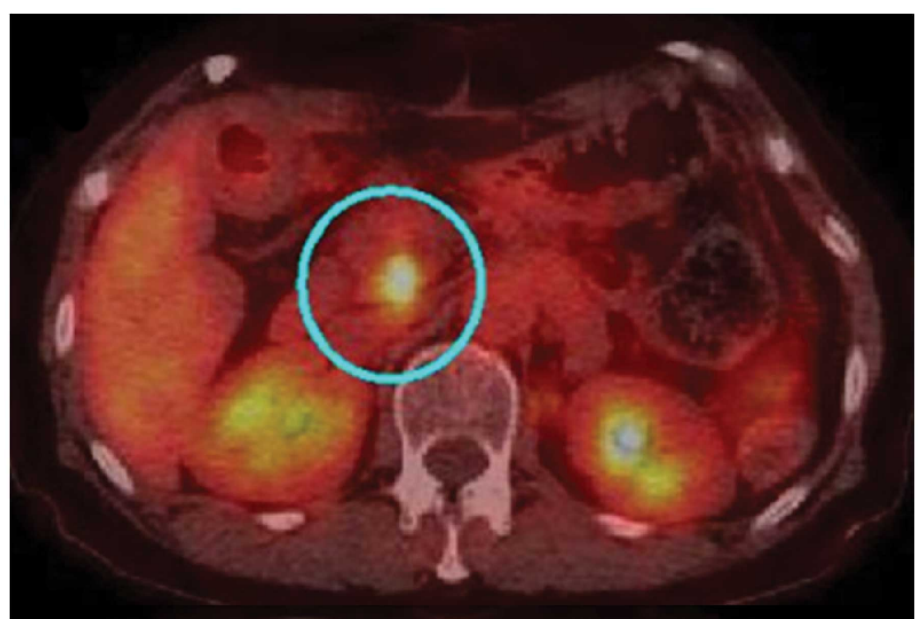

High-pressure situation. Like other tumors, this pancreatic cancer tumor, shown in a PET scan, may be subject to increasing pressure from all sides as it grows. Experiments suggest that this compression increases drug resistance.

Credit: H. Tajima et al., Oncol. Lett. 7, 1049 (2014) optimize chemotherapy treatments for a wide range of tumor conditions.

Hundreds of years ago, cancer treatments often involved physically compressing tumors, but these approaches were largely abandoned in the 19th century in favor of surgery and chemical treatments. Researchers now have a much better understanding of the ways by which mechanical forces can affect tumor growth and of the forces tumors generate naturally as they grow. "Most solid tumors grow in a confined space. It's like you're in a subway at rush hour and you want to expand your arms-you need to push into the surroundings in order to have your space," says Morgan Delarue, a biophysicist at the French National Centre for Scientific Research (CNRS) in Toulouse. Previous studies have shown that compressed tumors in cancer patients have limited blood flow, and a 2011 study found that compression could inhibit cell proliferation (see Viewpoint: Forcing Tumor Arrest).

Aware of these effects and of the way cancer drugs work, Delarue and his colleagues wondered how drug resistance might be affected by the compression that growing tumors experience. He says the question was this: "Knowing that [compressed] cells proliferate slower, and knowing that most drugs that we use to treat cancers target cells that do proliferate, what happens when you add the drug on cells that are under mechanical stress?"

To answer the question, the researchers used pancreatic cancer cells to construct dozens of "spheroids"-conglomerates of cells a few hundred micrometers in diameter that act like tumors. The team let some spheroids grow freely in a culture medium, while they placed others in agarose or dextran, which are gels 


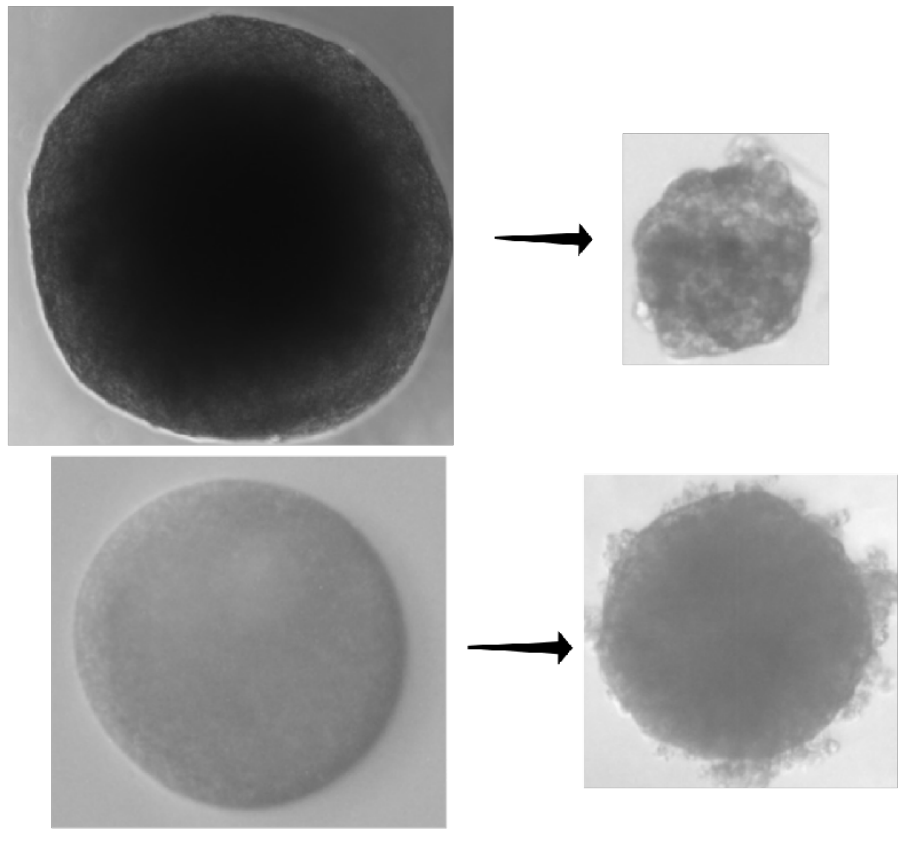

Performance under pressure. Researchers applied drugs to clumps of cancer cells grown in two different conditions-some had grown in an ordinary medium (top left), and others had grown in a viscous gel that compressed the clumps as they grew (bottom left). The drugs shrank the first category by 1.5 times more (top right) than the second category (bottom right).

Credit: M. Delarue/CNRS

that confined the growing spheroids, pressing back against the clumps as they expanded. After 8 days, the free-growing spheroids were roughly 2.5 times larger, but the confined spheroids had only enlarged by 1.5 times.

The researchers then applied a commonly used chemotherapeutic, either gemcitabine or docetaxel, to both sets of spheroids. These drugs target cells that are proliferating. After receiving a drug, free spheroids shrank 1.5 times more and ended up smaller than confined spheroids.

Next, Delarue and his colleagues constructed a simple model (a set of equations) that links spheroid size to cell proliferation rate. This model allowed them to predict the expected rate of cell death for a drug-exposed spheroid based on the amount of compression it experiences. Applying this formula to their data allowed the team to confirm that drug resistance could be explained by a compression-induced decrease in cell proliferation, rather than by other effects, such as reduced activation of the drug within the cell or a lack of drug uptake.

The results fit with other recent work suggesting that cancer researchers should be aware of the effects of tumor compression when designing drugs or prescribing treatment plans, Delarue says. The importance of pressure on drug resistance could also lead to novel treatments that involve reducing the compression of tumors. However, Delarue says that questions remain. For example, it's unclear why compression causes decreased proliferation or how the relationship between compression and proliferation varies among different kinds of tumors.

Some researchers would like to see more proof. "They need a lot more experiments to prove their principle," says Carman Ip, a biologist at the University of Chicago. "They can use, for example, different concentrations of the agarose" as a way to test the relationship between pressure, cell proliferation, and subsequent drug resistance.

However, the new findings align with other results involving mechanical forces, says Paolo Provenzano, a biomedical engineer at the University of Minnesota, Minneapolis. "It makes perfect sense that [tumors] would behave this way," but you still have to run the tests to find out. "It's a critical, important experiment," he says.

Dan Garisto is a freelance science writer based in New York.

\section{REFERENCES}

1. I. F. Rizzuti et al., "Mechanical control of cell proliferation increases resistance to chemotherapeutic agents," Phys. Rev. Lett. 125, 128103 (2020). 